Article

\title{
Proteomic Analysis of Whole Human Saliva Detects Enhanced Expression of Interleukin-1 Receptor Antagonist, Thioredoxin and Lipocalin-1 in Cigarette Smokers Compared to Non-Smokers
}

\author{
Kala Jessie ${ }^{1}$, Wei Wei Pang ${ }^{2}$, Zubaidah Haji Abdul Rahim ${ }^{1}$ and Onn Haji Hashim ${ }^{2,3, *}$ \\ 1 Department of Oral Biology, Faculty of Dentistry, University of Malaya, 50603 Kuala Lumpur, \\ Malaysia; E-Mails: jessiekala7@yahoo.com (K.J.); zubaidar@um.edu.my (Z.H.A.R.) \\ 2 University of Malaya Centre for Proteomics Research, Faculty of Medicine, University of Malaya, \\ 50603 Kuala Lumpur, Malaysia; E-Mail: wwpang@um.edu.my \\ 3 Department of Molecular Medicine, Faculty of Medicine, University of Malaya, 50603 Kuala \\ Lumpur, Malaysia \\ * Author to whom correspondence should be addressed; E-Mail: onnhashim@um.edu.my; \\ Fax: $+603-79674957$.
}

Received: 6 September 2010; in revised form: 25 October 2010 / Accepted: 5 November 2010 / Published: 9 November 2010

\begin{abstract}
A gel-based proteomics approach was used to screen for proteins of differential abundance between the saliva of smokers and those who had never smoked. Subjecting precipitated proteins from whole human saliva of healthy non-smokers to two-dimensional electrophoresis (2-DE) generated typical profiles comprising more than 50 proteins. While 35 of the proteins were previously established by other researchers, an additional 22 proteins were detected in the 2-DE saliva protein profiles generated in the present study. When the 2-DE profiles were compared to those obtained from subjects considered to be heavy cigarette smokers, three saliva proteins, including interleukin-1 receptor antagonist, thioredoxin and lipocalin-1, showed significant enhanced expression. The distribution patterns of lipocalin-1 isoforms were also different between cigarette smokers and non-smokers. The three saliva proteins have good potential to be used as biomarkers for the adverse effects of smoking and the risk for inflammatory and chronic diseases that are associated with it.
\end{abstract}


Keywords: saliva; proteome; smoker; biomarker

\section{Introduction}

Cigarette smoking is the most preventable cause of addiction, sickness and mortality in the world. Death attributed to cigarette smoking is estimated to rise from 5.4 million in 2005 to 6.4 million by 2015 [1]. Chronic cigarette smoking is the single most important risk factor for lung and oral cancers, cardiovascular diseases, chronic obstructive pulmonary disease (COPD) and other tobacco related oral diseases, including periodontitis [2-7]. Cigarette smoke contains more than 60 carcinogens and around 4,000 chemicals, including bacteria-derived endotoxins, which are toxic to cells [8-10]. The risk of developing tobacco smoking-related diseases increases with the total exposure time to the cigarette smoke, which generally includes the number of cigarettes a person smokes each day and the number of years a person has been smoking [11].

The oral cavity is the first organ in the human body to be exposed to the cigarette smoke. The tobacco smoke alters normal homeostasis of the oral cavity, including the saliva's antioxidant and other protective systems. This may lead to oral inflammatory diseases and oral cancers [12-15]. Early tumorigenic activities have been detected in normal oral mucosa of heavy smokers who have no overt precancerous or cancerous lesions [16]. The mucosal changes in smokers may also arise from the drying effects of the mucosa, high intraoral temperatures, intraoral $\mathrm{pH}$ changes, local alteration of membrane barriers and immune responses, or altered resistance to bacteria, fungal and viral infections. Smoking-related cell damage may leave molecular footprints in the saliva, offering the potential for non-invasive early diagnosis of tobacco-related oral diseases.

Human saliva contains a large number of proteins and peptides that are easily accessible and may serve as a potential source of biomarkers to monitor changes that occur under pathological conditions. The value of saliva as a biological fluid for the detection of diagnostic and prognostic biomarkers has become increasingly well established [17-24]. Collection of human saliva is a simple, non-invasive and cost-effective approach for screening large populations. It is easy to handle and may be repeated without inflicting much discomfort to the subjects [17,18].

Proteomic analysis is an important investigative tool used to systematically explore cellular proteins that are responsive to adverse environmental challenges. Several proteomic approaches, including those involving separation of proteins by two-dimensional electrophoresis (2-DE), have been applied in the investigation of biomarker candidates in the human saliva [25-29]. Recently, saliva has been shown to harbor potential informative biomarkers for oral cancer [30-32], head and neck cancer [33,34], and breast cancer [35]. While effects of the cigarette smoke on proteins expressed in the bronchoalveolar lavage [36-38], nasal lavage fluid [39], urine [40], lung tissue [41], bronchial airway epithelium and pooled exhaled breath condensate samples [42] have been analyzed, little information is available regarding the effects of smoking on the whole saliva proteome.

To the best of our knowledge, there had been no reported studies that specifically compared the expression of proteins in the saliva of smokers and non-smokers. In this study, 2-DE-based proteomics was used to screen for saliva proteins of differential abundance between smokers and subjects who had never smoked. The aberrantly expressed proteins, when correlated to those similarly altered in the 
saliva of patients with tobacco-related diseases including oral cancer, may potentially be used as biomarkers to indicate risks for the various diseases.

\section{Materials and Methods}

\subsection{Collection of Whole Saliva}

Unstimulated whole saliva samples were collected from 24 healthy Malay male volunteers aged between 35 and 55 years (12 smokers and 12 non-smokers), with no history of diabetes, autoimmune diseases or exposure to radiation and chemotherapy. Characteristics of the participants who were considered heavy smokers in this study are shown in Table 1. Saliva samples were collected with the volunteers' consent and approval granted by the Ethical committee (Institutional Review Board) of the Faculty of Dentistry, University of Malaya. Each subject answered a questionnaire concerning personal data, smoking and alcohol drinking habits, health or dental problems, oral hygiene habits, previous dental examinations, use of prescriptions, quantity and length of cigarette smoking. Unstimulated whole saliva was collected in the morning between 9 and 11 am to minimize the circadian effect, and subjects refrained from eating, drinking, smoking or performing any oral hygiene for at least $2 \mathrm{~h}$ prior to the collection. The difference between the mean flow rates of non-smokers $(0.39 \pm 0.04 \mathrm{~mL} / \mathrm{min})$ and smokers $(0.42 \pm 0.04 \mathrm{~mL} / \mathrm{min})$ was not statistically significant. Protease inhibitor cocktail was added to the saliva immediately after collection as previously described [43]. To remove debris and cells, the saliva was centrifuged at $14,000 \mathrm{~g}$ for $20 \mathrm{~min}$ at $4{ }^{\circ} \mathrm{C}$ and the proteins were precipitated in $10 \% \mathrm{TCA} /$ acetone $/ 20 \mathrm{mM}$ DTT. Saliva proteins were quantified using the Bradford protein assay kit (Bio-Rad, Hercules, USA) according to the manufacturer's instructions.

Table 1. Demographics and smoking history of smoker subjects.

\begin{tabular}{ccccc}
\hline Subject $^{(\mathbf{a})}$ & Age & Cigarettes/Day & Smoking Duration $^{(\mathbf{b})}$ & Stick-year $^{(\mathbf{c})}$ \\
\hline 1 & 35 & 20 & 15 & 300 \\
2 & 36 & 14 & 23 & 322 \\
3 & 38 & 20 & 15 & 300 \\
4 & 51 & 14 & 20 & 280 \\
5 & 36 & 14 & 15 & 210 \\
6 & 54 & 20 & 30 & 600 \\
7 & 38 & 20 & 17 & 340 \\
8 & 54 & 30 & 30 & 900 \\
9 & 39 & 20 & 20 & 400 \\
10 & 39 & 20 & 25 & 500 \\
11 & 48 & 24 & 17 & 408 \\
12 & 40 & 14 & 15 & 210 \\
\hline
\end{tabular}

(a) All subjects were male and of Malay ethnicity; ${ }^{(b)}$ Duration in years since first started smoking;

(c) Stick-year of exposure is in accordance to the Brickman index, which is the number of cigarettes smoked per day multiplied by the smoking duration. 


\subsection{Two-Dimensional Electrophoresis}

Two-dimensional electrophoresis (2-DE) was performed as previously described [43]. Saliva proteins $(130 \mu \mathrm{g})$ were dissolved in rehydration buffer containing $7 \mathrm{M}$ urea, $2 \mathrm{M}$ thiourea, 4\% CHAPS, 0.5\% IPG buffer, $65 \mathrm{mM}$ DTT and $0.002 \%$ bromophenol blue and applied onto $13 \mathrm{~cm}$ rehydrated precast immobilized drystrips $\mathrm{pH}$ 4-7 (GE Healthcare BioSciences, Uppsala, Sweden). Isoelectric focusing (IEF) for the first dimension and SDS-PAGE for the second dimension were performed as described previously [43]. All samples were analyzed in triplicate.

\subsection{Silver Staining}

The 2-DE gels were developed by silver staining as described by Heukeshoven and Dernick [44]. For mass spectrometry analyses, gels were stained with compatible silver staining with slight modifications according to Yan et al. [45].

\subsection{Image Analysis}

The LabScan image scanner was used to capture and store images of 2-DE gels. The GE ImageMaster $^{\mathrm{TM}} 2 \mathrm{D}$ Platinum Software version 7 was used to evaluate the protein profiles and perform protein analyses. To detect proteins that were differentially secreted in the saliva, the percentage volume contribution (\% vol) of a protein spot, which refers to the spot volume of a protein expressed as a percentage of the total spot volume of all detected saliva proteins, was calculated. Data expressed this way are independent of variations attributed to protein loading and staining. The 2-DE profiles and relative spot intensities obtained were reproducible when performed in triplicate.

\subsection{In Gel Trypsin Digestion and Mass Spectrometry}

Highly resolved protein spots were initially identified by visual comparison with previously published protein maps obtained from the human whole saliva [22-26]. The protein spots (1-2 mm diameter) were excised from silver-stained gels with pipette tips and kept hydrated in clean microfuge tubes containing Milli-Q water, prior to the in-gel digestion. Trypsin digestion and precise identification by mass spectrometry, using the MALDI-TOF/TOF instrument (Applied Biosystem 4800 Proteomic Analyzer), were performed as previously described [46].

\subsection{Database Searches}

Spectra were processed and analyzed by the Global Protein Server Workstation (Applied Biosystems), which uses the internal MASCOT (Matrix Science, London, UK) software for search of the peptide mass fingerprints and MS/MS data. Searches were performed against the Swiss-Prot database (Last update: October 23, 2008, containing 261513 sequences). Database search parameters were set as follows: The enzyme trypsin was used; up to one missed cleavage was allowed; variable modification included were carbamidomethylation of cysteine and oxidation of methionine; the mass tolerance for MS precursor ion and MS/MS fragment ion were $100 \mathrm{ppm}$ and $0.2 \mathrm{Da}$, respectively; and only monoisotopic masses were included in the search. 


\subsection{Statistical Analysis}

All values are presented as mean \pm S.E.M (standard error of the mean). The Student's t-test was used to analyze the significance of difference between non-smokers and smokers. The false discovery rate control was performed using the method of Benjamini and Hochberg [47].

\section{Results}

Figure 1 shows a typical 2-DE profile of saliva proteins separated between $\mathrm{pH} 4$ and 7 in healthy non-smokers. This range of $\mathrm{pH}$ was chosen as our earlier 2-DE results performed at a $\mathrm{pH}$ range of 3 to 10 showed that most of the saliva proteins were located in the acidic region between $\mathrm{pH} 4$ to 7 . More than 120 spots were detected in the whole saliva samples using the 2-DE that was performed under the conditions of our study. Identities of 108 spots belonging to 57 different proteins were established by MS and database search (Table 2). Some of these proteins, including polymeric immunoglobulin receptor (spots 3-9), carbonic anhydrase VI (spots 27-32), prolactin inducible proteins (spots 81-86), zinc-alpha-2-glycoprotein (spots 43 and 44), short palate, lung and nasal epithelium carcinoma-associated protein 1 (spots 58-61), cystatin S (spots 90 and 91) and lipocalin-1 (spots 87-89) were resolved in several isoforms and thus separated into distinct spots in the 2-DE gels.

Figure 1. Typical 2-DE profile of precipitated saliva proteins obtained from non-smokers. A total of 108 protein spots (circled and numbered) were identified by mass spectrometry and database search (please refer to Table 2). Acid side of 2-DE gel is to the left and relative molecular mass declines from the top.

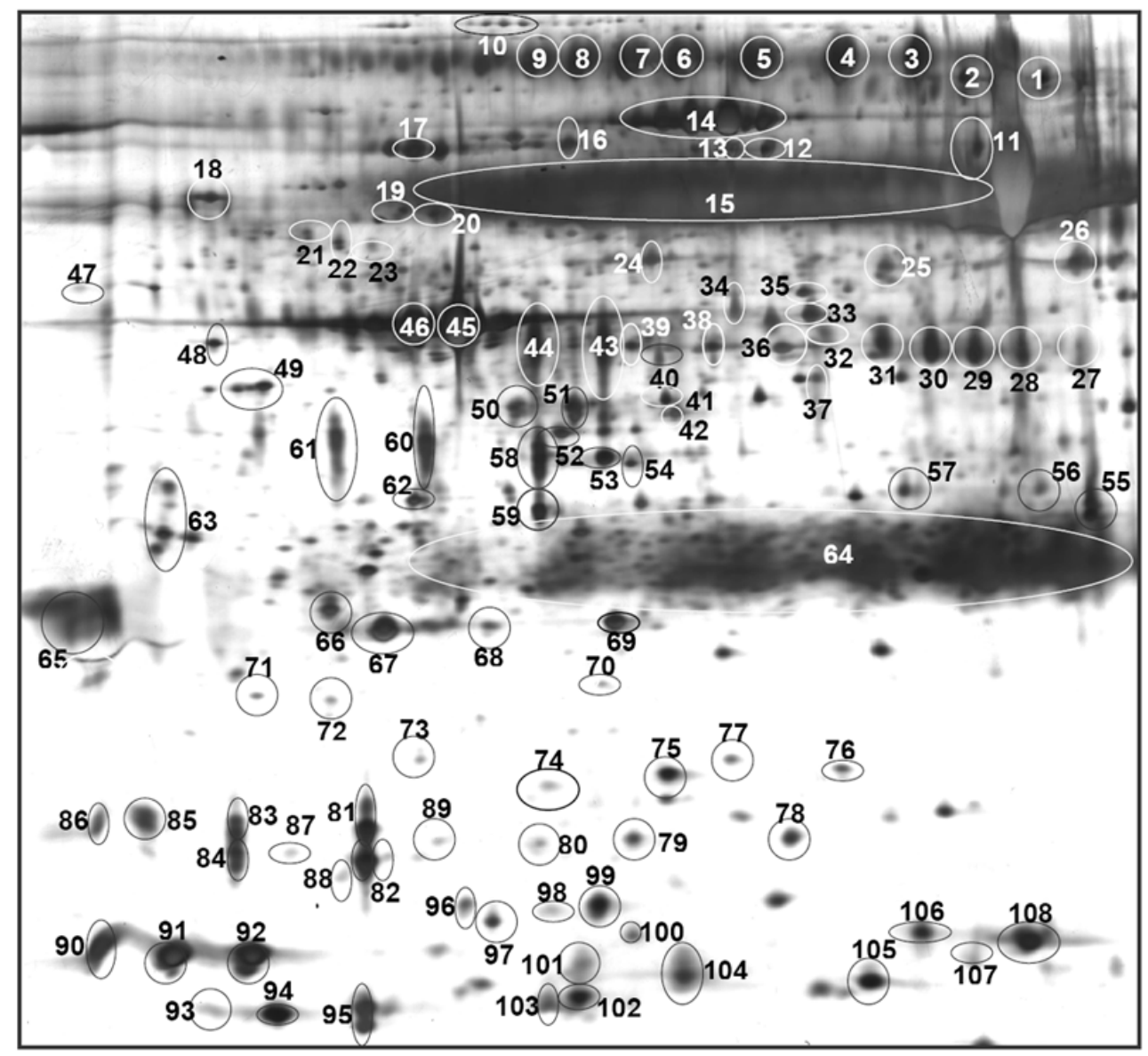


Table 2. Identification of saliva proteins by mass spectrometry.

\begin{tabular}{|c|c|c|c|c|c|c|}
\hline $\begin{array}{l}\text { Accession } \\
\text { No. }{ }^{\text {(a) }}\end{array}$ & Protein & $\begin{array}{l}\text { Spot } \\
\text { No. }{ }^{(b)}\end{array}$ & $\begin{array}{l}\text { MASCOT } \\
\text { Score }\end{array}$ & $\begin{array}{l}\text { No. of Peptides } \\
\text { Hit }\end{array}$ & $\begin{array}{l}\text { Sequence } \\
\text { Coverage }\end{array}$ & Ref. \\
\hline \multirow[t]{2}{*}{ P02787 } & Serotransferrin & 1 & 89 & 10 & 9 & {$[22,23,25]$} \\
\hline & & 2 & 78 & 16 & 8 & \\
\hline \multirow[t]{7}{*}{ P01833 } & Polymeric immunoglobulin & 3 & 282 & 9 & 8 & {$[22-26]$} \\
\hline & receptor & 4 & 111 & 11 & 33 & \\
\hline & & 5 & 99 & 17 & 27 & \\
\hline & & 6 & 240 & 7 & 22 & \\
\hline & & 7 & 246 & 20 & 55 & \\
\hline & & 8 & 169 & 12 & 32 & \\
\hline & & 9 & 120 & 18 & 28 & \\
\hline Q9Y6R7 & IgGFc-binding protein & 10 & 645 & 10 & 2 & $\mathrm{Npd}$ \\
\hline Q8TDL5 & $\begin{array}{l}\text { *Long palate, lung and nasal } \\
\text { epithelium carcinoma- } \\
\text { associated protein } 1\end{array}$ & 11 & 187 & 4 & 11 & Npd \\
\hline P09960 & *Leukotriene A-4 hydrolase & 12 & 376 & 6 & 14 & Npd \\
\hline \multirow[t]{2}{*}{ P02768 } & Serum albumin & 13 & 98 & 3 & 5 & [22-26] \\
\hline & & 14 & 122 & 5 & 7 & \\
\hline P04745 & Human salivay $\alpha$-amylase & 15 & 101 & 15 & 27 & [22-26] \\
\hline P08107 & Heat shock $70 \mathrm{kDa}$ protein 1 & 16 & 572 & 8 & 14 & [23] \\
\hline P13796 & Plastin-2 & 17 & 555 & 32 & 16 & Npd \\
\hline P07237 & *Protein disulfide-isomerase & 18 & 636 & 13 & 27 & $\mathrm{Npd}$ \\
\hline \multirow[t]{2}{*}{ P02774 } & Vitamin D-binding protein & 19 & 741 & 11 & 32 & [27] \\
\hline & precursor & 20 & 575 & 10 & 27 & \\
\hline \multirow[t]{3}{*}{ P01009 } & Alpha-1-antitrypsin & 21 & 211 & 8 & 10 & [25] \\
\hline & & 22 & 112 & 6 & 12 & \\
\hline & & 23 & 99 & 5 & 18 & \\
\hline P61158 & Actin-related protein 3 & 24 & 327 & 13 & 17 & Npd \\
\hline P50395 & $\begin{array}{l}\text { *Rab GDP dissociation } \\
\text { inhibitor beta }\end{array}$ & 25 & 363 & 15 & 19 & [28] \\
\hline P06733 & Alpha-enolase & 26 & 735 & 11 & 33 & {$[22,23,26]$} \\
\hline \multirow[t]{6}{*}{$\mathrm{P} 23280$} & Carbonic anhydrase VI & 27 & 290 & 15 & 19 & {$[22,23]$} \\
\hline & & 28 & 90 & 4 & 4 & \\
\hline & & 29 & 303 & 11 & 40 & \\
\hline & & 30 & 488 & 32 & 48 & \\
\hline & & 31 & 79 & 4 & 8 & \\
\hline & & 32 & & & & \\
\hline \multirow[t]{2}{*}{ P30740 } & *Leukocyte elastase inhibitor & 33 & 374 & 12 & 19 & [29] \\
\hline & & 34 & 315 & 10 & 16 & \\
\hline Q99536 & $\begin{array}{l}\text { **Synaptic vesicle membrane } \\
\text { protein VAT- } 1\end{array}$ & 35 & 76 & 2 & 4 & Npd \\
\hline P40121 & Macrophage-capping protein & 36 & 579 & 16 & 20 & $\mathrm{Npd}$ \\
\hline
\end{tabular}


Table 2. Cont.

\begin{tabular}{|c|c|c|c|c|c|c|}
\hline \multirow[t]{3}{*}{ P02675 } & \multirow[t]{3}{*}{ Fibrinogen beta chain } & 37 & 676 & 12 & 26 & \multirow[t]{3}{*}[23,25]{} \\
\hline & & 38 & 554 & 16 & 36 & \\
\hline & & 39 & 467 & 15 & 20 & \\
\hline P00738 & Haptoglobin & 40 & 518 & 10 & 21 & {$[28]$} \\
\hline \multirow[t]{2}{*}{ P37837 } & \multirow[t]{2}{*}{ *Transaldolase } & 41 & 60 & 8 & 15 & \multirow[t]{2}{*}[28]{} \\
\hline & & 42 & 77 & 6 & 13 & \\
\hline \multirow[t]{2}{*}{ P25311 } & \multirow[t]{2}{*}{ Zinc-alpha-2-glycoprotein } & 43 & 246 & 16 & 28 & \multirow[t]{2}{*}[22-26]{} \\
\hline & & 44 & 285 & 11 & 20 & \\
\hline \multirow[t]{2}{*}{ P60709 } & \multirow[t]{2}{*}{ Actin, cytoplasmic 1} & 45 & 230 & 3 & 15 & \multirow[t]{2}{*}[23,25]{} \\
\hline & & 46 & 188 & 4 & 15 & \\
\hline P27797 & $* *$ Calreticulin & 47 & 651 & 10 & 27 & Npd \\
\hline \multirow[t]{2}{*}{ P01024 } & \multirow[t]{2}{*}{ Complement C3 } & 48 & 330 & 15 & 6 & \multirow[t]{2}{*}{ Npd } \\
\hline & & 49 & 332 & 14 & 5 & \\
\hline P63261 & Actin, cytoplasmic 2 & 50 & 285 & 11 & 20 & {$[23]$} \\
\hline P60709 & Actin, cytoplasmic 1 & 51 & 243 & 5 & 14 & {$[23,25]$} \\
\hline P52907 & $\begin{array}{l}\text { F-actin-capping protein subunit } \\
\text { alpha-1 }\end{array}$ & 52 & 274 & 6 & 16 & Npd \\
\hline P25311 & Zinc-alpha-2-glycoprotein & 53 & 82 & 3 & 10 & {$[22-26]$} \\
\hline P12429 & Annexin A3 & 54 & 331 & 8 & 14 & Npd \\
\hline P00738 & Haptoglobin & 55 & 627 & 12 & 24 & {$[28]$} \\
\hline P01876 & Ig alpha-1 chain $\mathrm{C}$ region & 56 & 274 & 5 & 17 & {$[22-24,26]$} \\
\hline P30740 & *Leukocyte elastase inhibitor & 57 & 417 & 20 & 30 & {$[29]$} \\
\hline \multirow[t]{4}{*}{ Q96DR5 } & \multirow{4}{*}{$\begin{array}{l}\text { *Short palate, lung and nasal } \\
\text { epithelium carcinoma-associated } \\
\text { protein } 2\end{array}$} & 58 & 141 & 4 & 15 & \multirow[t]{4}{*}[22]{} \\
\hline & & 59 & 119 & 5 & 18 & \\
\hline & & 60 & 293 & 20 & 68 & \\
\hline & & 61 & & & & \\
\hline O00299 & $\begin{array}{l}* \text { Chloride intracellular channel } \\
\text { protein } 1\end{array}$ & 62 & 561 & 9 & 52 & Npd \\
\hline P63104 & $\begin{array}{l}\text { Protein kinase } C \text { inhibitor } \\
\text { protein-1(14-3-3 protein } \\
\text { zeta/delta) }\end{array}$ & 63 & 101 & 4 & 12 & Npd \\
\hline P01834 & Ig kappa chain $\mathrm{C}$ region & 64 & 101 & 4 & 33 & {$[23-26]$} \\
\hline P01591 & Immunoglobulin J chain & 65 & 241 & 11 & 32 & {$[22,24,25]$} \\
\hline P52565 & $\begin{array}{l}\text { Rho GDP-dissociation } \\
\text { inhibitor } 1\end{array}$ & 66 & 356 & 13 & 30 & Npd \\
\hline P52566 & $\begin{array}{l}* \text { Rho GDP-dissociation } \\
\text { inhibitor } 2\end{array}$ & 67 & 173 & 9 & 31 & Npd \\
\hline \multirow[t]{2}{*}{ P09211 } & \multirow[t]{2}{*}{ Glutathione S Transferase } & 68 & 201 & 6 & 22 & \multirow[t]{2}{*}[22-24,26]{} \\
\hline & & 69 & 493 & 19 & 61 & \\
\hline P32119 & Peroxiredoxin-2 & 70 & 264 & 6 & 27 & {$[28]$} \\
\hline \multirow[t]{2}{*}{ P02763 } & \multirow[t]{2}{*}{ Alpha-1-acid glycoprotein 1} & 71 & 317 & 6 & 27 & \multirow[t]{2}{*}{ Npd } \\
\hline & & 72 & 137 & 4 & 17 & \\
\hline P18510 & $\begin{array}{l}\text { Interleukin-1 receptor antagonist } \\
\text { protein }\end{array}$ & 73 & 148 & 6 & 15 & {$[22,24]$} \\
\hline
\end{tabular}


Table 2. Cont.

\begin{tabular}{|c|c|c|c|c|c|c|}
\hline \multirow[t]{3}{*}{ P00738 } & Haptoglobin & 74 & 358 & 6 & 22 & \multirow[t]{3}{*}[28]{} \\
\hline & & 75 & 491 & 7 & 20 & \\
\hline & & 76 & 439 & 8 & 22 & \\
\hline P15531 & $\begin{array}{l}* * \text { Nucleoside diphosphate } \\
\text { kinase } \mathrm{A}\end{array}$ & 77 & 132 & 6 & 25 & Npd \\
\hline P52566 & $\begin{array}{l}\text { Rho GDP-dissociation } \\
\text { inhibitor } 2\end{array}$ & 78 & 185 & 4 & 32 & Npd \\
\hline O15511 & $\begin{array}{l}\text { Actin-related protein } 2 / 3 \\
\text { complex subunit } 5\end{array}$ & 79 & 69 & 2 & 7 & Npd \\
\hline \multirow[t]{7}{*}{$\mathrm{P} 12273$} & \multirow[t]{7}{*}{ Prolactin-inducible protein } & 80 & 385 & 6 & 45 & \multirow[t]{7}{*}[22-25]{} \\
\hline & & 81 & 116 & 32 & 6 & \\
\hline & & 82 & 375 & 5 & 44 & \\
\hline & & 83 & 365 & 6 & 45 & \\
\hline & & 84 & 410 & 6 & 45 & \\
\hline & & 85 & 413 & 7 & 54 & \\
\hline & & 86 & 279 & 5 & 45 & \\
\hline \multirow[t]{3}{*}{ P31025 } & \multirow[t]{3}{*}{ *Lipocalin-1 } & 87 & 169 & 5 & 18 & \multirow[t]{3}{*}[22,24]{} \\
\hline & & 88 & 168 & 3 & 17 & \\
\hline & & 89 & 213 & 4 & 21 & \\
\hline \multirow[t]{2}{*}{ P01036 } & \multirow[t]{2}{*}{${ }^{*}$ Cystatin S } & 90 & 535 & 15 & 62 & \multirow[t]{2}{*}[22-24,26]{} \\
\hline & & 91 & 444 & 8 & 58 & \\
\hline P09228 & ${ }^{*}$ Cystatin SA & 92 & 437 & 7 & 64 & {$[22-26]$} \\
\hline \multirow[t]{2}{*}{ P10599 } & \multirow[t]{2}{*}{ Thioredoxin } & 93 & 88 & 4 & 16 & \multirow[t]{2}{*}[21]{} \\
\hline & & 94 & 111 & 3 & 15 & \\
\hline P12273 & Prolactin-inducible protein & 95 & 413 & 7 & 54 & {$[22-25]$} \\
\hline P02766 & Transthyretin & 96 & 80 & 3 & 33 & {$[28,33]$} \\
\hline Q14019 & $* *$ Coactosin-like protein & 97 & 379 & 8 & 60 & Npd \\
\hline P08118 & $* *$ Beta-microseminoprotein & 98 & 119 & 2 & 8 & Npd \\
\hline P02766 & Transthyretin & 99 & 269 & 7 & 40 & {$[28,33]$} \\
\hline Q01469 & $\begin{array}{l}\text { *Fatty acid-binding protein, } \\
\text { epidermal }\end{array}$ & 100 & 219 & 6 & 47 & {$[22-24,29]$} \\
\hline P01036 & ${ }^{*}$ Cystatin $\mathrm{S}$ & 101 & 192 & 4 & 37 & {$[22-24,26]$} \\
\hline P06702 & Calgranulin-B & 102 & 296 & 6 & 51 & {$[22,24,26]$} \\
\hline P01040 & Cystatin-A & 103 & 42 & 1 & 18 & {$[22,25,26]$} \\
\hline P06702 & Calgranulin-B & 104 & 395 & 7 & 63 & {$[22,24,26]$} \\
\hline P28325 & ${ }^{*}$ Cystatin D & 105 & 172 & 6 & 34 & {$[22,24]$} \\
\hline Q01469 & $\begin{array}{l}\text { *Fatty acid-binding protein, } \\
\text { epidermal }\end{array}$ & 106 & 347 & 8 & 52 & {$[22-24,29]$} \\
\hline Q05315 & **Eosinophil lysophospholipase & 107 & 108 & 3 & 9 & Npd \\
\hline P01037 & ${ }^{*}$ Cystatin SN & 108 & 293 & 20 & 68 & {$[22-26]$} \\
\hline
\end{tabular}

(a) Accession no. are in accordance to Swiss-Prot; ${ }^{\text {(b) }}$ Spot numbers are those referred to in Figures 1 and 2 and identified by MS/MS; Npd - proteins not previously detected in the saliva proteome using 2-DE; * Proteins found only in saliva and not in plasma; ** Proteins detected for the first time in the saliva proteome of this study. 
Among the total of 57 saliva proteins, 35 had been previously identified using 2-DE [32-39], whereas 16, including plastin-2, actin-related protein-3, C3 complement precursor, macrophage capping protein, $\mathrm{F}$ actin capping protein, annexin A3, protein kinase $\mathrm{C}$ inhibitor protein-1, rho-GDP-dissociation inhibitor 1, rho-GDP-dissociation inhibitor 2, actin-related protein 2/3 complex subunit 5, alpha-1-acid glycoprotein 1, chloride intracellular channel protein 1, protein disulfide-isomerase, leukotriene A-4 hydrolase, IgGFc-binding protein and long palate, lung and nasal epithelium carcinoma-associated protein 1, were previously detected using liquid-based proteomics [21,23]. The other six saliva proteins, eosinophil lysophospholipase, beta-microseminoprotein, coactosin-like protein, nucleoside diphosphate kinase A, calreticulin and synaptic vesicle membrane protein VAT-1, are reported for the first time by this study.

When 2-DE was performed on whole saliva samples of heavy smokers, the profiles obtained were similar to those from non-smokers. All 57 different proteins that were expressed in the saliva of the non-smokers were also detected in the saliva of the heavy smokers although the rates of presence of 16 proteins in the 2-DE profiles of the cigarette smokers were different from those of the non-smokers. When the 2-DE protein profiles obtained from the non- and heavy smokers were subjected to densitometry analysis, initially a significantly enhanced expression of seven proteins including polymeric immunoglobulin receptor, complement $\mathrm{C} 3$, $\alpha 1$-antitrypsin, calgranulin $\mathrm{B}$, interleukin-1 receptor antagonist, thioredoxin and lipocalin-1, was detected between the two subject groups. However, only three of the proteins, i.e., interleukin-1 receptor antagonist $(+3$ fold $)$, thioredoxin $(+2.5$ fold $)$ and lipocalin-1 ( +4.4 fold) were found to be truly significant when the $p$-values were corrected for false significant results using the method of Benjamini and Hochberg [47] (Table 3). Figure 2 demonstrates examples of 2-DE spot clusters of proteins whose levels were altered in the saliva obtained from the heavy smokers as compared to those of the non-smokers.

Table 3. Densitometry analysis of saliva proteins and their rates of presence in 2-DE profiles.

\begin{tabular}{|c|c|c|c|c|c|}
\hline \multirow[b]{2}{*}{ Protein } & \multicolumn{2}{|c|}{ Non-smokers } & \multicolumn{2}{|c|}{ Smokers } & \multirow[b]{2}{*}{$p^{(c)}$} \\
\hline & $\begin{array}{c}\text { \% volume }^{(a)} \\
( \pm \text { S.E.M) }\end{array}$ & $\mathbf{R P}^{(\mathbf{b})} / \mathbf{1 2}$ & $\begin{array}{c}\text { \% volume }^{(\mathrm{a})} \\
( \pm \text { S.E.M) }\end{array}$ & $\mathbf{R P} \mathbf{P}^{(\mathbf{b})} / \mathbf{1 2}$ & \\
\hline \multicolumn{6}{|l|}{ 1: Energy/Metabolism } \\
\hline Amylase & $14.15( \pm 0.54)$ & 12 & $12.72( \pm 0.85)$ & 12 & 0.168 \\
\hline Carbonic anhydrase VI & $1.48( \pm 0.17)$ & 12 & $1.07( \pm 0.14)$ & 12 & 0.077 \\
\hline Zinc-alpha-2-glycoprotein & $1.02( \pm 0.09)$ & 12 & $1.09( \pm 0.13)$ & 12 & 0.673 \\
\hline $\begin{array}{l}\text { Fatty acid-binding protein, } \\
\text { epidermal }\end{array}$ & $0.08( \pm 0.01)$ & 12 & $0.12( \pm 0.02)$ & 12 & 0.111 \\
\hline Transaldolase & $0.04( \pm 0.01)$ & 12 & $0.04( \pm 0.01)$ & 12 & 0.543 \\
\hline Alpha-enolase & $0.06( \pm 0.02)$ & 10 & $0.10( \pm 0.02)$ & 12 & 0.183 \\
\hline \multicolumn{6}{|l|}{ 2: Defence/Immune response } \\
\hline $\begin{array}{l}\text { Polymeric immunoglobulin } \\
\text { receptor }\end{array}$ & $4.58( \pm 0.12)$ & 12 & $3.57( \pm 0.40)$ & 12 & 0.024 \\
\hline Immunoglobulin $\mathrm{J}$ chain & $0.38( \pm 0.06)$ & 12 & $0.41( \pm 0.06)$ & 12 & 0.305 \\
\hline $\begin{array}{l}\text { Interleukin-1 receptor antagonist } \\
\text { protein }\end{array}$ & $0.01( \pm 0.00)$ & 7 & $0.04( \pm 0.01)$ & 11 & 0.004 \\
\hline
\end{tabular}


Table 3. Cont.

\begin{tabular}{|c|c|c|c|c|c|}
\hline Prolactin-inducible protein & $2.27( \pm 0.20)$ & 12 & $2.35( \pm 0.23)$ & 12 & 0.787 \\
\hline $\begin{array}{l}\text { Short palate, lung and nasal } \\
\text { epithelium carcinoma-associated } \\
\text { protein } 2\end{array}$ & $1.22( \pm 0.18)$ & 11 & $1.42( \pm 0.18)$ & 12 & 0.456 \\
\hline Alpha-1-acid glycoprotein 1 & $0.01( \pm 0.00)$ & 2 & $0.02( \pm 0.01)$ & 5 & 0.159 \\
\hline \multicolumn{6}{|l|}{ 3: Protein degradation inhibitor } \\
\hline$\alpha_{1}$-Antitrypsin & $0.02( \pm 0.01)$ & 6 & $0.05( \pm 0.01)$ & 8 & 0.027 \\
\hline Cystatin A & $0.08( \pm 0.02)$ & 12 & $0.09( \pm 0.03)$ & 12 & 0.620 \\
\hline Cystatin S & $0.04( \pm 0.01)$ & 12 & $0.05( \pm 0.01)$ & 12 & 0.775 \\
\hline Cystatin SA & $0.28( \pm 0.05)$ & 12 & $0.27( \pm 0.07)$ & 12 & 0.916 \\
\hline Cystatin SN & $0.28( \pm 0.06)$ & 12 & $0.30( \pm 0.04)$ & 12 & 0.744 \\
\hline Cystatin D & $0.12( \pm 0.02)$ & 12 & $0.12( \pm 0.02)$ & 11 & 0.769 \\
\hline Leukocyte elastase inhibitor & $0.10( \pm 0.01)$ & 12 & $0.10( \pm 0.01)$ & 12 & 0.922 \\
\hline \multicolumn{6}{|l|}{ 4: Cell adhesion/communication } \\
\hline Calgranulin B & $0.13( \pm 0.01)$ & 12 & $0.21( \pm 0.04)$ & 12 & 0.032 \\
\hline \multicolumn{6}{|l|}{ 5: Protein folding/repair } \\
\hline Heat shock $70 \mathrm{kDa}$ protein 1 & $0.06( \pm 0.01)$ & 12 & $0.05( \pm 0.01)$ & 12 & 0.818 \\
\hline \multicolumn{6}{|l|}{ 6: Redox } \\
\hline Thioredoxin & $0.03( \pm 0.00)$ & 1 & $0.07( \pm 0.02)$ & 8 & 0.001 \\
\hline Peroxiredoxin-2 & $0.01( \pm 0.01)$ & 3 & $0.02( \pm 0.01)$ & 7 & 0.617 \\
\hline \multicolumn{6}{|l|}{ 7: Signaling } \\
\hline Complement $\mathrm{C} 3$ precursor & $0.00( \pm 0.00)$ & 3 & $0.01( \pm 0.00)$ & 9 & 0.012 \\
\hline Glutathione-S Transferase & $0.10( \pm 0.01)$ & 12 & $0.12( \pm 0.02)$ & 12 & 0.444 \\
\hline Rho GDP-dissociation inhibitor 1 & $0.14( \pm 0.03)$ & 3 & $0.15( \pm 0.03)$ & 3 & 0.557 \\
\hline Rho GDP-dissociation inhibitor 2 & $0.03( \pm 0.01)$ & 10 & $0.04( \pm 0.01)$ & 12 & 0.327 \\
\hline $\begin{array}{l}\text { Protein kinase } \mathrm{C} \text { inhibitor } \\
\text { protein-1 }\end{array}$ & $0.11( \pm 0.03)$ & 7 & $0.17( \pm 0.05)$ & 11 & 0.282 \\
\hline Annexin A3 & $0.07( \pm 0.02)$ & 11 & $0.00( \pm 0.01)$ & 6 & 0.088 \\
\hline \multicolumn{6}{|l|}{ 8: Structural/cytoskeletal } \\
\hline $\begin{array}{l}\text { F-actin-capping protein subunit } \\
\text { alpha-1 }\end{array}$ & $0.02( \pm 0.01)$ & 4 & $0.03( \pm 0.01)$ & 8 & 0.521 \\
\hline Macrophage-capping protein & $0.03( \pm 0.01)$ & 7 & $0.02( \pm 0.00)$ & 9 & 0.261 \\
\hline L-plastin & $0.11( \pm 0.01)$ & 12 & $0.09( \pm 0.01)$ & 12 & 0.376 \\
\hline \multicolumn{6}{|l|}{ 9: Transport } \\
\hline Lipocalin-1 & $0.15( \pm 0.05)$ & 8 & $0.65( \pm 0.13)$ & 12 & 0.001 \\
\hline Haptoglobin & $0.06( \pm 0.01)$ & 11 & $0.07( \pm 0.01)$ & 12 & 0.694 \\
\hline Transthyretin & $0.07( \pm 0.01)$ & 12 & $0.10( \pm 0.02)$ & 12 & 0.108 \\
\hline Serum albumin & $1.63( \pm 0.14)$ & 12 & $1.71( \pm 022)$ & 12 & 0.758 \\
\hline
\end{tabular}

${ }^{\text {(a) }}$ volume of a protein expressed as a percentage of the total spot volume of all proteins; ${ }^{(b)}$ rate of presence of the protein spots in the 12 2-DE profiles that were analyzed; ${ }^{(c)} p$-values of less than $0.0068(p<0.0068)$ were considered statistically significant when the false discovery rate procedure of Benjamini and Hochberg [47] was performed to the data set. 
Figure 2. Cropped images of saliva proteins in the 2-DE gels of non-smokers and smokers. Representative gels of two differentially expressed saliva proteins are shown. Spot numbers are those referred to in Table 2.

\section{Non-smoker}

\section{IL-1 receptor antagonist}
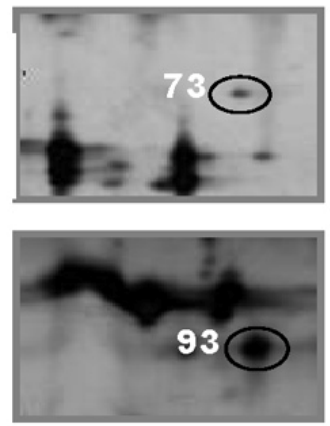
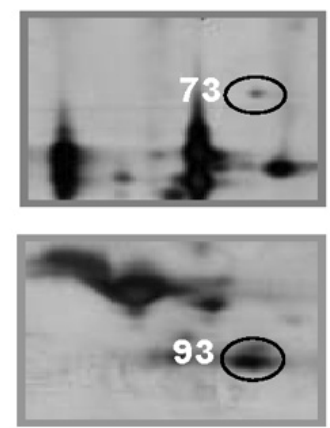

Smoker
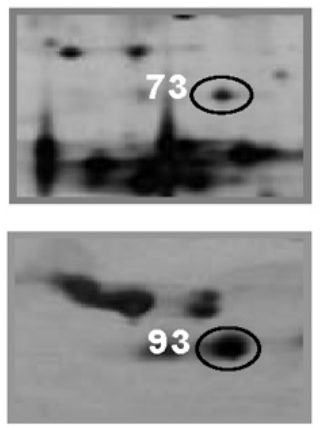
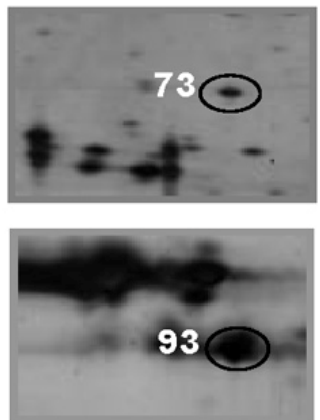

When the different isoforms of polymeric immunoglobulin receptor (spots 3-9), carbonic anhydrase VI (spots 27-32), prolactin inducible proteins (spots 81-86), zinc-alpha-2-glycoprotein (spots 43 and 44), short palate, lung and nasal epithelium carcinoma-associated protein 1 (spots 58-61) and cystatin S (spots 90 and 91) were similarly analyzed by densitometry, their volume distribution patterns were found to be consistent between the saliva of non-smokers and smokers. In contrast, the 2-DE volume distribution pattern for isoforms of lipocalin-1 in the saliva of non-smokers was different from that detected in the saliva of the heavy smokers (Figure 3). Among the seven isoforms analyzed, the isoform $\mathrm{f}$ was almost exclusive to the saliva of the smokers (Table 4).

Figure 3. Cropped images of lipocalin-1 isoform spots in the 2-DE gels of non-smokers and smokers. Six representative gels are shown. The isoform spots a to $g$ are marked in the gels (only represented in one of the images so as not to affect image display). Detailed densitometry analysis of the isoform spots is demonstrated in Table 4.

Non-smoker
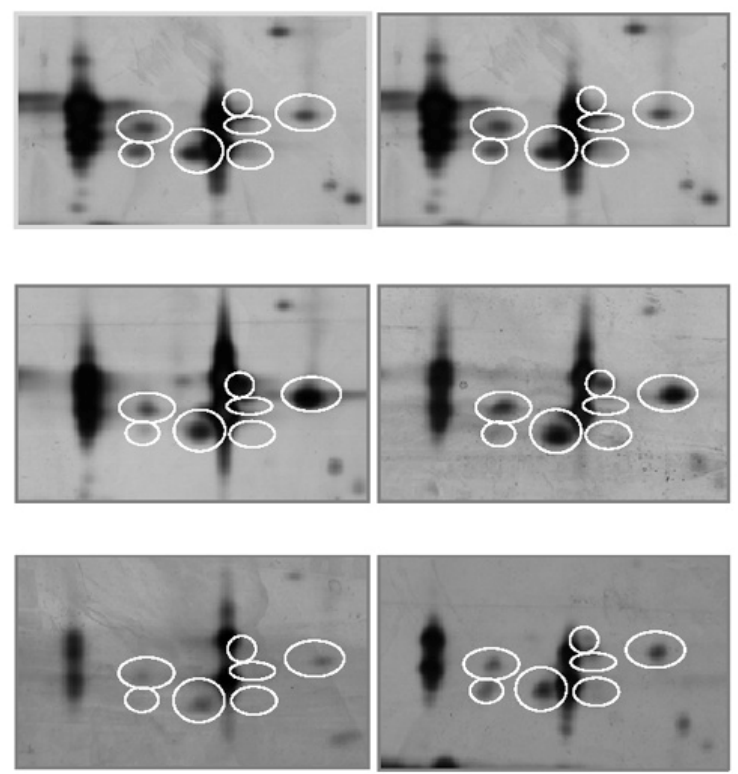

Smoker
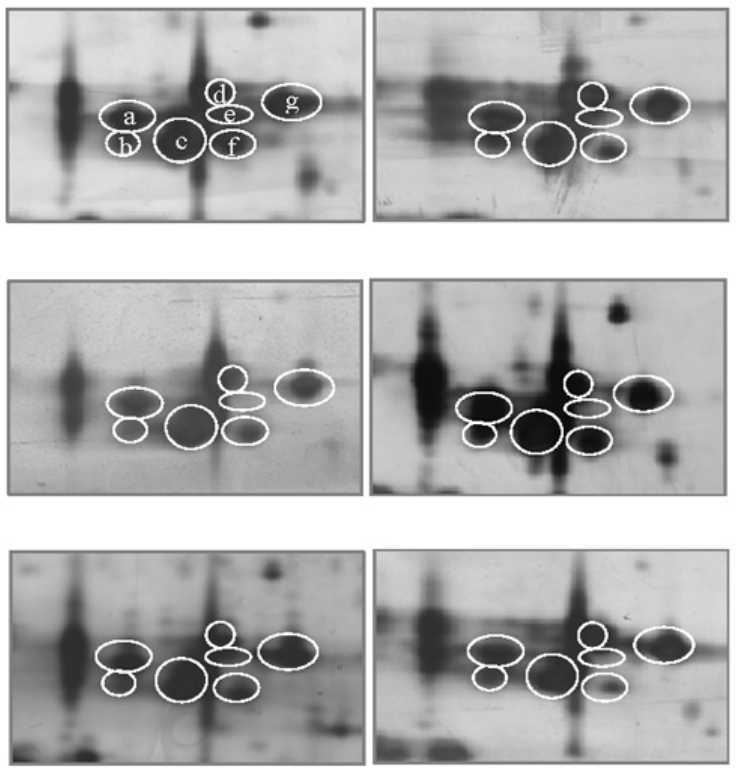
Table 4. Densitometry analysis of lipocalin-1 isoforms and their rates of presence in 2-DE profiles.

\begin{tabular}{|c|c|c|c|c|c|c|}
\hline \multirow{2}{*}{$\begin{array}{l}\text { Isoform } \\
\text { Spot }^{(a)}\end{array}$} & \multicolumn{2}{|c|}{ Non-smokers } & \multicolumn{2}{|c|}{ Smokers } & \multirow[b]{2}{*}{$p$} & \multirow{2}{*}{$\begin{array}{c}\text { Fold } \\
\text { Change }^{(d)}\end{array}$} \\
\hline & $\%$ vol $^{(b)}$ & $\mathbf{R P}^{(\mathbf{c})}$ & $\%$ vol $^{(b)}$ & $\mathbf{R P}^{(\mathbf{c})}$ & & \\
\hline $\mathrm{a}$ & 0.056 & 8 & 0.195 & 12 & 0.005 & +3.5 \\
\hline $\mathrm{b}$ & 0.007 & 4 & 0.150 & 10 & 0.000 & +21.4 \\
\hline $\mathrm{c}$ & 0.085 & 11 & 0.305 & 12 & 0.012 & +3.7 \\
\hline $\mathrm{d}$ & 0.013 & 5 & 0.108 & 11 & 0.012 & +8.3 \\
\hline $\mathrm{e}$ & 0.006 & 4 & 0.069 & 9 & 0.038 & +11.5 \\
\hline $\mathrm{f}$ & 0.003 & 1 & 0.089 & 6 & 0.031 & +29.7 \\
\hline $\mathrm{g}$ & 0.110 & 12 & 0.316 & 12 & 0.018 & +2.9 \\
\hline
\end{tabular}

(a) isoforms of lipocalin-1 as depicted in Figure $3 ;{ }^{(b)}$ volume of a protein expressed as a percentage of the total spot volume of all proteins; ${ }^{(c)}$ rate of presence of the protein spots in the $122-\mathrm{DE}$ profiles that were analyzed; ${ }^{(d)}$ fold change is the ratio of \%vol of smokers to non-smokers.

\section{Discussion}

Human whole saliva contains fluid from the salivary glands, gingival crevicular fluid, bronchiol and nasal secretions, desquamated epithelial cells, oral tissues, and very often, the components of blood, bacteria and viruses [48-50]. Therefore, whole saliva - in contrast to serum - is a hostile environment with proteins subjected to the effects of many host- and bacteria-derived enzymes. Some saliva proteins are synthesized in the salivary glands and subsequently subjected to intracellular processing including glycosylation, phosphorylation and proteolysis. Once the secretions enter the non-sterile oral environment, additional and continuous protein modifications by host- and bacteria-derived enzymes occur. This results in the possible generation of many modified proteins in whole saliva [51].

The 2-DE profiles of proteins in whole saliva from healthy non-smokers that were generated in the present study showed strong resemblance to those that were previously reported [22-26]. Almost 90\% of the protein spots that were highly resolved were eventually identified. The remaining spots were unidentifiable as the proteins generated low intensity spectra probably due to their low amounts, resistance to proteolytic cleavage, low recovery of digested peptides, and/or low efficiency in peptide ionization. Nevertheless, it is also possible that some of the unidentified proteins were of bacterial origin since the mouth is likely to harbor a lot of microorganisms.

In addition to the 35 human saliva proteins that have previously been established by other research groups using 2-DE [22-26], the present study detected the presence of 22 additional proteins. This is an important contribution to the human saliva proteome as a whole. Among the newly identified proteins (see Table 2), nucleotide diphosphate kinase A, annexin A3, Rho-GDP-dissociation inhibitor 1, beta-microseminoprotein, chloride intracellular channel protein 1, protein disulfide-isomerase, calreticulin, peroxiredoxin-2, alpha-1-acid glycoprotein 1 and IgG Fc-binding protein are considered clinically interesting as they have been previously associated with cancer and other diseases [52-61].

The establishment of highly resolved 2-DE protein profiles enabled investigations on protein changes associated with cigarette smoking. Densitometry analyses on the 2-DE protein profiles obtained from the non- and heavy smokers showed differential abundance of interleukin-1 receptor antagonist, thioredoxin and lipocalin-1 between the saliva samples of the two subject groups (Table 3). The three proteins have good potential to be used as non-specific complementary biomarkers for the 
adverse effects of smoking although this requires further evaluation and correlative studies. Some of the proteins may be used as risk indicators for inflammatory and chronic diseases that are associated with smoking as they have been shown to be of increased levels in the saliva of the patients. In the case of lipocalin-1, the isoform distribution pattern detected was also found to differ between smokers and non-smokers. This suggests that the carbohydrate moieties of lipocalin-1 of the heavy cigarette smokers were different from those of the non-smokers and that they may be differently glycosylated or modified. However, this remains to be further established.

Despite being distinctly categorized according to their primary biological roles [62], the three saliva proteins that were altered in abundance reflect the body's overall response to the damaging effects of heavy smoking. The high levels of IL-1 receptor antagonist in the saliva of the heavy smokers detected in this study reflect an anti-inflammatory response in the oral cavities of the smokers. Increased generation of the proteins in smokers may be induced by the proinflammatory cytokines that were promoted by oxidative stress [63-65]. An imbalance between IL-1 receptor antagonist and IL-1 has been hypothesized to play a role in the pathogenesis of various inflammatory diseases [65].

Lipocalin-1 and thioredoxin are proteins most likely involved in the response to stress in relation to tissue damage. The high levels of lipocalin-1 and thioredoxin in the cigarette smokers' saliva may reflect their function as an oxidative stress-induced scavenger against toxic and pro-inflammatory lipids [66-68]. Lipocalin-1 had been suggested to be a cysteine proteinase inhibitor [67] and may have a role in the control of inflammatory processes in oral tissues. Thioredoxin, on the other hand, was shown to modulate remodeling factors in response to the cigarette smoke [68]. Increased secretion of thioredoxin had been previously demonstrated in the saliva of patients with oral cancer [32].

\section{Conclusion}

Comparative proteomics analysis of human saliva samples from subjects who were considered heavy cigarette smokers and those who did not smoke detected altered abundance of interleukin-1 receptor antagonist, thioredoxin and lipocalin-1, as well as a change in the isoform distribution patterns of lipocalin-1. These proteins may be used as early biomarkers to indicate risks of tobacco-related diseases.

\section{Acknowledgements}

This work was funded by research grants PS105/2008A and PS071/2009A from the University of Malaya. We are grateful to Karuthan Chinna and Jaime Jacqueline Jayapalan for their kind help with the statistical analysis.

\section{References}

1. Fellows, J.L.; Trosclair, A.; Adams, E.K.; Rivera, C.C. Annual smoking-attributable mortality, year of potential life lost, and economics costs-United States, 1995-1999. Morbidity Mortality Wkly. Rep. 2002, 51, 300-303.

2. Freedman, N.D.; Leitzmann, M.F.; Hollenbeck, A.R.; Schatzkin, A.; Abnet, C.C. Cigarette smoking and subsequent risk of lung cancer in men and women: analysis of a prospective cohort study. Lancet Oncol. 2008, 9, 649-656. 
3. Ko, Y.C.; Huang, Y.L.; Lee, C.H.; Chen, M.J.; Lin, L.M.; Tsai, C.C. Betel quid chewing, cigarette smoking and alcohol consumption related to oral cancer in Taiwan. J. Oral Pathol. Med. 1995, 24, 450-453.

4. Zheng, T.; Holford, T.; Chen, Y.; Jiang, P.; Zhang, B.; Boyle, P. Risk of tongue cancer associated with tobacco smoking and alcohol consumption. Oral Oncol. 1997, 33, 82-85.

5. Ockene, I.S.; Miller, N.H. Cigarette smoking, cardiovascular disease, and stroke: a statement for healthcare professionals from the American Heart Association. American Heart Association Task Force on Risk Reduction. Circulation 1997, 96, 3243-3247.

6. Yoshida, T.; Tuder, R.M. Pathobiology of cigarette smoke-induced chronic obstructive pulmonary disease. Physiol. Rev. 2007, 87, 1047-1082.

7. Bergstrom, J. Tobacco smoking and chronic destructive periodontal disease. Odontology 2004, 92, 1-8.

8 Pauly, J.L.; Waight, J.D.; Paszkiewicz, G.M. Tobacco flakes on cigarette filters grow bacteria: A potential health risk to the smoker? Tob. Control 2008, 17, i49-i52.

9. Tobacco smoke and involuntary smoking. In IARC Monographs on the Evaluation of Carcinogenic Risks to Humans, Lyon, France, 11-18 June, 2002; Volume 83.

10. Hasday, J.D.; Bascom, R.; Costa, J.J.; Fitzgerald, T.; Dubin, W. Bacterial endotoxin is an active component of cigarette smoke. Chest 1999, 115, 829-835.

11. Lubin, J.H.; Purdue, M.; Kelsey, K.; Zhang, Z.F.; Winn, D.; Wei, Q.; Talamini, R.; Szeszenia-Dabrowska, N.; Sturgis, E.M.; Smith, E.; et al. Total exposure and exposure rate effects for alcohol and smoking and risk of head and neck cancer: a pooled analysis of case-control studies. Epidemiology 2009, 170, 937-947.

12. Hershkovich, O.; Oliva, J.; Nagler, R.M. Lethal synergistic effect of cigarette smoke and saliva in an in vitro model: Does saliva have a role in the development of oral cancer? Eur. J. Cancer 2004, 40, 1760-1767.

13. Nagler, R.M.; Reznick, A.Z. Cigarette smoke effects on salivary antioxidants and oral cancernovel concepts. Isr. Med. Assoc. J. 2004, 6, 691-694.

14. Nagler, R.; Dayan, D. The dual role of saliva in oral carcinogenesis. Oncology 2006, 71, 10-17.

15. Reznick, A.Z.; Klein, I.; Eiserich, J.P.; Cross, C.E.; Nagler, R.M. Inhibition of oral peroxidase activity by cigarette smoke: in vivo and in vitro studies. Free Radic. Biol. Med. 2003, 34, 377-384.

16. Ayan, N.; Ayan, I.; Alatli, C.; Guler, S.D.; Dalkilic, C.; Cinar, F.; Dogan, O. P53 overexpression in normal oral mucosa of heavy smokers. J. Exp. Clin. Cancer Res. 2000, 19, 525-529.

17. Wong, D.T. Salivary diagnostics for oral cancer. J. Calif. Dent. Assoc. 2006, 34, 303-308.

18. Hofman, L.F. Human saliva as diagnostic specimen. J. Nutr. 2001, 131, 1621S-1625S.

19. Slavkin, H.C. Toward molecular based diagnostic for the oral cavity. J. Am. Dent. Assoc. 1998, 129, 1138-1143.

20. Ferguson, D.B. Saliva as a diagnostic fluid. Acta Stomatologia Croat. 2009, 43, 77-82.

21. Xie, H.; Rhodus, N.L.; Griffin, R.J.; Carlis, J.V.; Griffin, T.J. A catalogue of human saliva proteins identified by free flow electrophoresis-based peptide separation and tandem mass spectrometry. Mol. Cell. Proteomics 2005, 4, 1826-1830. 
22. Vitorino, R.; Lobo, M.J.; Ferrer-Correira, A.J.; Dubin, J.R.; Tomer, K.B.; Domingues, P.M.; Amado, F.M. Identification of human whole saliva protein components using proteomics. Proteomics 2004, 4, 1109-1115.

23. Hu, S.; Xie, Y.; Ramachandran, P.; Ogorzalek Loo, R.R.; Li, Y.; Loo, J.A.; Wong, D.T. Large-scale identification of proteins in human salivary proteome by liquid chromatography/mass spectrometry and two-dimensional gel elcetrophoresis-mass spectrometry. Proteomics 2005, 5, 1714-1728.

24. Ghafouri, B.; Tagesson, C.; Lindahl, M. Mapping of proteins in human saliva using twodimensional gel electrophoresis and peptide mass fingerprinting. Proteomics 2003, 3, 1003-1015.

25. Huang, C.M. Comparative proteomic analysis of human whole saliva. Arch. Oral Biol. 2004, 49, 951-962.

26. Hirtz, C.; Chevalier, F.; Centeno, D.; Egea, J.; Rossignol, M.; Sommerer, N.; de Périère, D. Complexity of the human whole saliva proteome. J. Physiol. Biochem. 2005, 61, 469-480.

27. Wu, Y.; Shu, R.; Luo, L.J.; Ge, L.H.; Xie, Y.F. Initial comparison of proteomic profiles of whole unstimulated saliva obtained from generalized aggressive periodontitis patients and healthy control subjects. J. Periodontal Res. 2009, 44, 636-644.

28. Haigh, B.J.; Stewart, K.W.; Whelan, J.R.; Barnett, M.P.; Smolenski, G.A.; Wheeler, T.T. Alterations in the salivary proteome associated with periodontitis. J. Clin. Periodontol. 2010, 37, 241-247.

29. Giusti, L.; Baldini, C.; Bazzichi, L.; Ciregia, F.; Tonazzini, I.; Mascia, G.; Giannaccini, G.; Bombardieri, S.; Lucacchini, A. Proteome analysis of whole saliva: a new tool for rheumatic diseases - the example of Sjögren's syndrome. Proteomics 2007, 7, 1634-1643.

30. Hu, S.; Arellano, M.; Boontheung, P.; Wang, J.; Zhou, H.; Jiang, J.; Elashoff, D.; Wei, R.; Loo, J.A.; Wong, D.T. Salivary proteomic for oral cancer biomarker discovery. Clin. Cancer Res. 2008, 14, 6246-6252.

31. Li, Y.; John, M.A., St.; Zhou, X.; Kim, Y.; Sinha, U.; Jordan, R.C.; Eisele, D.; Abemayor, E.; Elashoff, D.; Park, N.H.; Wong, D.T. Salivary transcriptome diagnostic for oral cancer detection. Clin. Cancer Res. 2004, 10, 8442-8450.

32. Hu, S.; Yu, T.; Xie, Y.; Yang, Y.; Li, Y.; Zhou, X.; Tsung, S.; Loo, R.R.; Loo, J.R.; Wong, D.T. Discovery of oral fluid biomarkers for human oral cancer by mass spectrometry. Cancer Genomics Proteomics 2007, 4, 55-64.

33. Dowling, P.; Wormald, R.; Meleady, P.; Henry, M.; Curran, A.; Clynes, M. Analysis of the saliva proteome from patients with head and neck squamous cell carcinoma reveals differences in abundance levels of proteins associated with tumour progression and metastasis. J. Proteomics 2008, 71, 168-175.

34. Ohshiro, K.; Rosenthal, D.I.; Koomen, J.M.; Streckfus, C.F.; Chambers, M.; Kobayashi, R.; El-Naggar, A.K. Pre-analytic saliva processing affect proteomic results and biomarker screening of head and neck squamous carcinoma. Int. J. Oncol. 2007, 30, 743-749.

35. Streckfus, C.; Bigler, L.; Tucci, M.; Thigpen, J.T. A preliminary study of cA15-3, c-erbB-2, epidermal growth factor receptor, cathepsin-D and p53 in saliva among women with breast carcinoma. Cancer Invest. 2000, 18, 101-109. 
36. Lindahl, M.; Ekstrom, T.; Sorensen, J.; Tagesson, C. Two dimensional protein patterns of bronchoalveolar lavage fluid from non-smokers, smokers, and subjects exposed to asbestos. Thorax 1996, 51, 1028-1035.

37. Plymoth, A.; Yang, Z.; Lofdahl, C.G.; Ekberg-Jansson, A.; Dahlback, M.; Fehniger, T.E.; Marko-Varga, G.; Hancock, W.S. Rapid proteome analysis of bronchoalveolar lavage samples of lifelong smokers and never smokers by micro-scale liquid chromatography and mass spectrometry. Clin. Chem. 2006, 52, 671-679.

38. Plymoth, A.; Lofdahl, C.G.; Ekberg-Jansson, A.; Dahlback, M.; Broberg, P.; Foster, M.; Fehniger, T.E.; Marko-Varga, G. Protein expression patterns associated with progression of chronic obstructive pulmonary disease in bronchoalveolar lavage of smokers. Clin. Chem. 2007, 53, 636-644.

39. Ghafouri, B.; Stahlbom, B.; Tagesson, C.; Lindahl, M. Newly identified proteins in human nasal lavage fluid from non-smokers and smokers using two-dimensional gel electrophoresis and peptide mass fingerprinting. Proteomics 2002, 2, 112-120.

40. Airoldi, L.; Magagnotti, C.; Iannuzzi, A.R.; Marelli, C.; Bagnati, R.; Pastorelli, R.; Colombi, A.; Santaguida, S.; Chiabrando, C.; Schiarea, S.; Fanelli, R. Effects of cigarette smoking on the human urinary proteome. Biochem. Biophys. Res. Commun. 2009, 381, 397-402.

41. Kelsen, S.G.; Duan, X.; Ji, R.; Perez, O.; Liu, C.; Merali, S. Cigarette smoke induced in unfold protein response in the human lung. Am. J. Respir. Cell. Mol. Biol. 2008, 38, 541-550.

42. Gianazza, E.; Allegra, L.; Bucchioni, E.; Eberini, I.; Puglisi, L.; Blasi, F.; Terzano, C.; Wait, R.; Sirtori, C.R. Increassed keratin content detected by proteomic analysis of exhaled breath condensate from healthy persons who smoke. Am. J. Med. 2004, 117, 51-54.

43. Jessie, K.; Hashim, O.H.; Rahim, Z.H.A. Precipitation methods for salivary proteins and rehydration buffer for two-dimensional electrophoresis. Biotechnology 2008, 7, 686-693.

44. Heukeshoven, J.; Dernick, R. Improved silver staining procedure for fast staining in PhastSystem Development Unit. I. Staining of sodium dodecyl sulfate gels. Electrophoresis 1988, 9, 28-32.

45. Yan, J.X.; Wait, R.; Berkelman, T.; Harry, R.A.; Westbrook, J.A.; Wheeler, C.H.; Dunn, M.J. A modified silver staining protocol for visualization of proteins compatible with matrix-assisted laser desorption/ionization and electrospray ionization-mass spectrometry. Electrophoresis 2000, 21, 3666-3672.

46. Seriramalu, R.; Pang, W.W.; Jayapalan, J.J.; Mohamed, E.; Abdul-Rahman, P.S.; Bustam, A.Z.; Khoo, A.S.B.; Hashim, O.H. Application of champedak mannose-binding lectin in the glycoproteomic profiling of serum samples unmasks reduced expression of alpha-2 macroglobulin and complement factor B in patients with nasopharyngeal carcinoma. Electrophoresis 2010, 31, 2388-2395.

47. Benjamini, Y.; Hochberg, Y. Controlling the false discovery rate: a practical and powerful approach to multiple testing. J. R. Stat. Soc. B 1995, 57, 289-300.

48. Humphrey, S.P.; Williamson, R.T. A review of saliva: normal composition, flow, and function. J. Prosthet. Dent. 2001, 85, 162-169.

49. Turner, R.J.; Sugiya, H. Understanding salivary fluid and protein secretion. Oral Dis. 2002, 8, $3-11$. 
50. Defabianis, P.; Re, F. The role of saliva in maintaining oral health. Minerva Stomatol. 2003, 52, 301-308.

51. Helmerhorst, E.J.; Oppenheim, F.G. Saliva: a dynamic proteome. Critical Reviews in Oral Biology and Medicine. J. Dent. Res. 2007, 86, 680-693.

52. Liu, Y.F.; Xiao, Z.Q.; Li, M.X.; Li, M.Y.; Zhang, P.F.; Li, C.; Li, F.; Chen, Y.H.; Yi, H.; Yao, H.X.; Chen, Z.C. Quantitative proteome analysis reveals annexin A3 as a novel biomarker in lung adenocarcinoma. J. Pathol. 2009, 217, 54-64.

53. Petrova, D.T.; Asif, A.R.; Armstrong, V.W.; Dimova, I.; Toshev, S.; Yaramov, N.; Oellerich, M.; Toncheva, D. Expression of chloride intracellular channel protein 1 (CLIC1) and tumor protein D52 (TPD52) as potential biomarkers for colorectal cancer. Clin. Biochem. 2008, 41, 1224-1236.

54. Gromov, P.; Gromova, I.; Bunkenborg, J.; Cabezon, T.; Moreira, J.M.; Timmermans-Wielenga, V.; Roepstorff, P.; Rank, F.; Celis, J.E. Up-regulated proteins in the fluid bathing the tumour cell microenvironment as potential serological markers for early detection of cancer of the breast. Mol. Oncol. 2010, 4, 65-89.

55. Wang, Z.; Feng, X.; Liu, X.; Jiang, L.; Zeng, X.; Ji, N.; Li, J.; Li, L.; Chen, Q. Involvement of potential pathways in malignant transformation from oral leukoplakia to oral squamous cell carcinoma revealed by proteomic analysis. BMC Genomics 2009, 10, 383.

56. Gupta, N.; Shankernarayan, N.P.; Dharmalingam, K. Alpha1-acid glycoprotein as a putative biomarker for monitoring the development of the type II reactional stage of leprosy. J. Med. Microbiol. 2010, 59, 400-407.

57. Almgren, M.A.E.; Henriksson, K.C.E.; Fujimoto, J.; Chang, C.L. Nucleoside diphosphate kinase $\mathrm{A} / \mathrm{nm} 23-\mathrm{H} 1$ promotes metastasis of NB69-derived human neuroblastoma. Mol. Cancer Res. 2004, 2, 387-394.

58. Allard, L.; Burkhard, P.R.; Lescuyer, P.; Burgess, J.A.; Walter, N.; Hochstrasser, D.F.; Sanchez, J.C. PARK7 and nucleoside diphosphate kinase A as plasma markers for the early diagnosis of stroke. Clin. Chem. 2005, 51, 2043-2051.

59. Lo, W.Y.; Tsai, M.H.; Tsai, Y.; Hua, C.H.; Tsai, F.J.; Huang, S.Y.; Tsai, C.H.; Lai, C.C. Identification of over-expressed proteins in oral squamous cell carcinoma (OSCC) patients by clinical proteomic analysis. Clin. Chim. Acta 2007, 376, 101-107.

60. O'Donovan, N.; Fischer, A.; Abdo, E.M.; Simon, F.; Peter, H.J.; Gerber, H.; Buergi, U.; Marti, U. Differential expression of IgG Fc binding protein (FcgammaBP) in human normal thyroid tissue, thyroid adenomas and thyroid carcinomas. J. Endocrinol. 2002, 174, 517-524.

61. Whitaker, H.C.; Warren, A.Y.; Eeles, R.; Kote-Jarai, Z.; Neal, D.E. The potential value of microseminoprotein-beta as a prostate cancer biomarker and therapeutic target. Prostate 2010, 70, 333-340.

62. Esser, D.; Alvarez-Llamas, G.A.; de Vries, M.P.; Weening, D.; Vonk, R.J.; Roelofsen, H. Sample Stability and Protein Composition of Saliva: Implications for Its Use as a Diagnostic Fluid. Biomark Insights 2008, 3, 25-37.

63. Patti, G.; D'Ambrosio, A.; Mega, S.; Giorgi, G.; Zardi, E.M.; Zardi, D.M.; Dicuonzo, G.; Dobrina, A.; Di Sciascio, G. Early interleukin-1 receptor antagonist elevation in patients with acute myocardial infraction. Journal of the American College of Cardiology. J. Am. Coll. Cardiol. 2004, 43, 35-38. 
64. Fearon, W.F.; Fearon, D.T. Inflammation and cardiovascular disease, the role of the interleukin-1 receptor antagonist. Circulation 2008, 117, 2577-2579.

65. Casini-Raggi, V.; Kam, L.; Chong, Y.J.; Fiocchi, C.; Pizarro, T.T.; Cominelli, F. Mucosal imbalance of IL-1 and IL-1 receptor antagonist in inflammatory bowel disease. a novel mechanism of chronic intestinal inflammation. J. Immunol. 1995, 154, 2434-2440.

66. Wojnar, P.; Dirnhofer, S.; Ladurner, P.; Berger, P.; Redl, B. Human lipocalin 1, a physiological scavenger of lipophilic compounds, is produced by corticotrophs of the pituitary gland. J. Histochem. Cytochem. 2002, 50, 433-435.

67. van't Hof, W.; Blankenvoorde, M.F.J.; Veerman, E.C.I.; Amerongen, A.V.N. The salivary lipocalin von Ebner's gland protein is a cysteine proteinase inhibitor. J. Biol. Chem. 1997, 272, 1837-1841.

68. Huang, Y.L.; Chuang, C.Y.; Sung, F.C.; Chen, C.Y. Thioredoxin overexpression modulates remodeling factors in stress responses to cigarette smoke. J. Toxicol. Environ. Health A 2008, 71, 1490-1498.

(C) 2010 by the authors; licensee MDPI, Basel, Switzerland. This article is an open access article distributed under the terms and conditions of the Creative Commons Attribution license (http://creativecommons.org/licenses/by/3.0/). 